\title{
Pick Your Poison: The Attribution Paradox in Cyberwar
}

\author{
Eric Jardine* and Nathaniel Porter \\ Virginia Tech \\ *corresponding author: ejardine@vt.edu
}

Draft. Please do not cite or distribute without permission of the authors

(Feb 26, 2020)

\begin{abstract}
Certainty matters in cyberspace. The so-called attribution problem can impede deterrence, making cyberattacks more likely. But attribution problems can also limit conflict escalation, keeping nations safer. Using a discrete choice experimental design, this article quantifies the scope of the attribution problem for both deterrence and escalation. The results suggest that the attribution problem is really only a problem for deterrence and actually helps limit conflict escalation by reducing support for more severe retaliatory options.
\end{abstract}


Cyberattacks against the United States happen daily. Data breaches abound, often compromising hundreds of millions of personal records [1]. Distributed denial of service attacks target websites, knocking them offline, rendering their services unusable, and costing both time and money to restore. Ransomware attacks lock whole cities out of their public information technology infrastructure, and impede, as in the case of The WannaCry ransomware attack in 2017, timely access to health and other public services. In 2018, the US Department of Homeland Security's National Cyber Awareness System even issued an alert indicating Russian actors had made plays in cyberspace toward US critical national infrastructure. These moves targeted, in the words of the alert itself, "the energy, nuclear, commercial facilities, water, aviation, and critical manufacturing sectors" [2]. Responding to these trends, numerous fevered calls have emerged, portending a coming cyber war, "Cyber Pearl Harbor," or "Cyber 9/11" [3, 4].

Yet, despite a mounting flurry of cyberattacks targeting individuals, private sector organizations, and public sector actors, full-blown cyberwar has not yet taken place [5-7]. In cyberspace, two empirical trends stand out in clear relief. First, cyber deterrence routinely fails. Second, increasingly frequent and damaging cyberattacks (but see [8-10]), do not seem to cue off pronounced escalatory cycles leading to greater conflict or conflagration. Why?

\section{Janus-Faced Attribution Problems}

The frequent failure of deterrence in cyberspace is well-documented, at least at the level of theory. In major ways, deterrence fails because of what is known as the "attribution problem" [11-17]. Stockpiled arsenals of guns, bombs, and cyber bullets can sometimes effectively deter opponents. Deterrence works through a process of backwards induction. Potential aggressors can be deterred through the threat of punishment if they think the costs associated with the probability that they will be identified and retaliated against outweigh the perceived benefits of launching an attack in the first place [16, 18, 19]. Attribution problems in cyberspace can stymie deterrence by punishment by making it hard to say who launched an initial attack.

In cyberspace, several factors can impede effective attribution. Technical design features of the Internet itself make tracing the source of an attack challenging, particularly if adversaries undertake necessary obfuscation steps [15]. Malicious actors might also work via arm's length cyber mercenaries to ensure plausible deniability [20]. Beyond these methods, attribution of a cyberattack also remains an inherently political problem [11,16], with clear legal and public opinion dimensions [21]. In short, the attribution problem implies uncertainty about "who done it" [11], allowing bad actors to get away with theft, disruption, degradation of service, and, perhaps someday, murder.

Yet, the attribution problem in cyberspace is both a matter of degree and more Janus-faced than is commonly realized. Empirical knowledge of the foundation of cyberwar is only recently beginning to be fleshed out [7, 12]. But, past literature has not yet determined what threshold of attribution is necessary for the public to support retaliatory options in the event of a cyberattack against the United States. Nor has the problem of attribution been clearly linked to the other readily demonstrable empirical trend in cyberspace: the relative absence of escalation from an initial cyberattack to increasingly severe response options such as military force. 
Using a discrete choice experimental design and US-based respondents, we empirically document just how much certainty about 'who done it' matters for public support of both any retaliatory moves against a possible aggressor (the deterrence side of the coin) and selection of more severe retaliatory options (the escalation side of things). The data show that attribution both cuts and heals, harms and helps. Low levels of certainty about who launched a hypothetical cyberattack on the US significantly reduce the likelihood that people support any retaliatory option, including a number of cross-domain foreign policy tools such as naming and shaming and the imposition of economic sanctions to in-kind cyber responses, or the use of military force [22-24]. Conversely, low levels of certainty also disproportionately inhibit support for more severe retaliatory choices, preventing conflicts from escalating from an initial attack into something bigger. Attribution problems in cyberspace, in other words, are both encouraging attacks and preventing serious conflagration.

\section{Data and Methods}

We collected the data to quantify the potential bidirectional scope and effect of attribution problems in cyberspace using a discrete choice experimental design. This data collection technique is extensively used as a preference elicitation technique in marketing research. Discrete choice experiments help to determine how much various attributes (variables) matter in people's decision-making process [25, 26]. 2,081 respondents were randomly assigned to one of three initial scenarios. These scenarios depicted a hypothetical cyberattack against the United States. The scenarios were otherwise identical, except for the details of how severe the attack was in terms of economic damage and loss of life. The scenarios ranged from the attack being narrowly prevented to a multi-state blackout costing billions of dollars and killing hundreds.

Following initial randomization into a framing scenario, respondents proceeded onto a page displaying a discrete choice table. Respondents were told that the government wanted to know if, and against whom, they would support retaliation for the initial attack depicted in the opening scenario. After making a choice, respondents were presented with another randomized discrete choice setup. Each respondent evaluated a total of five experimental setups before answering a set of demographic and attitudinal questions.

The pool of possible aggressor countries includes two US allies (UK and Israel), two US adversaries (Russia and China), and one more non-aligned state (India). For each potential aggressor, the table summarized five additional attributes of potential relevance to the decision of whether to retaliate: 1 ) the certainty with which the government says they know who launched the initial attack against the US; 2) the estimated probability that current inaction by the US will lead to another incoming attack; 3) the proposed means of retaliation, ranging from naming and shaming to economic sanctions, in-kind cyber retaliation, and the use of military force; 4) the estimated potential economic cost of retaliation; and 5) the estimated human cost of retaliation. Both the potential perpetrators and the attribute levels randomly varied in each experimental iteration. In total, 1,653 respondents completed the survey and passed the attention check. This sample population indicated their possible retaliation decisions for 8,265 pairs of potential aggressors. 
Analysis proceeded in 3 stages. We first used Average Marginal Component-specific Effects (AMCE) to estimate causal effects of each experimental factor on the probability of selecting a given retaliation option [25]. Next, we calculated the mean empirical probability of selecting a given option across values of individual experimental factors at each level of certainty. These models help to unpack how much the attribution problem (i.e. certainty with which the perpetrator is identified) affects deterrence by inhibiting or promoting retaliation against an identified aggressor. Finally, to answer how attribution and willingness to escalate to a costlier response interact, we used logistic regression on option selection to estimate the relative costbenefits ratios given the possible perpetrator, likelihood of another attack if there is no retaliation, and cost in dollars and lives lost of the proposed retaliation option, all interacted with the type of retaliation (name and shame, economic sanctions, in-kind cyber-attack, or military force). We split cases into deciles by this ratio and compared empirical selection probabilities across severity and certainty deciles.

\section{Results}

The experimental results suggest that certainty about 'who done it' is a crucial factor in the public's decision to support retaliation against a potential aggressor. When faced with a clear attack against the United States and then asked if they would support retaliation against one of two tentatively identified potential aggressors, most people weighed the degree of certainty about the perpetrator quite highly in making their choice. Figure 1 plots AMCE estimated causal effects of each experimental condition. While higher certainty about 'who done it' typically results in a higher predicted probability of choosing to retaliate as oppose to doing nothing, there are really three clusters in the plotted results. 

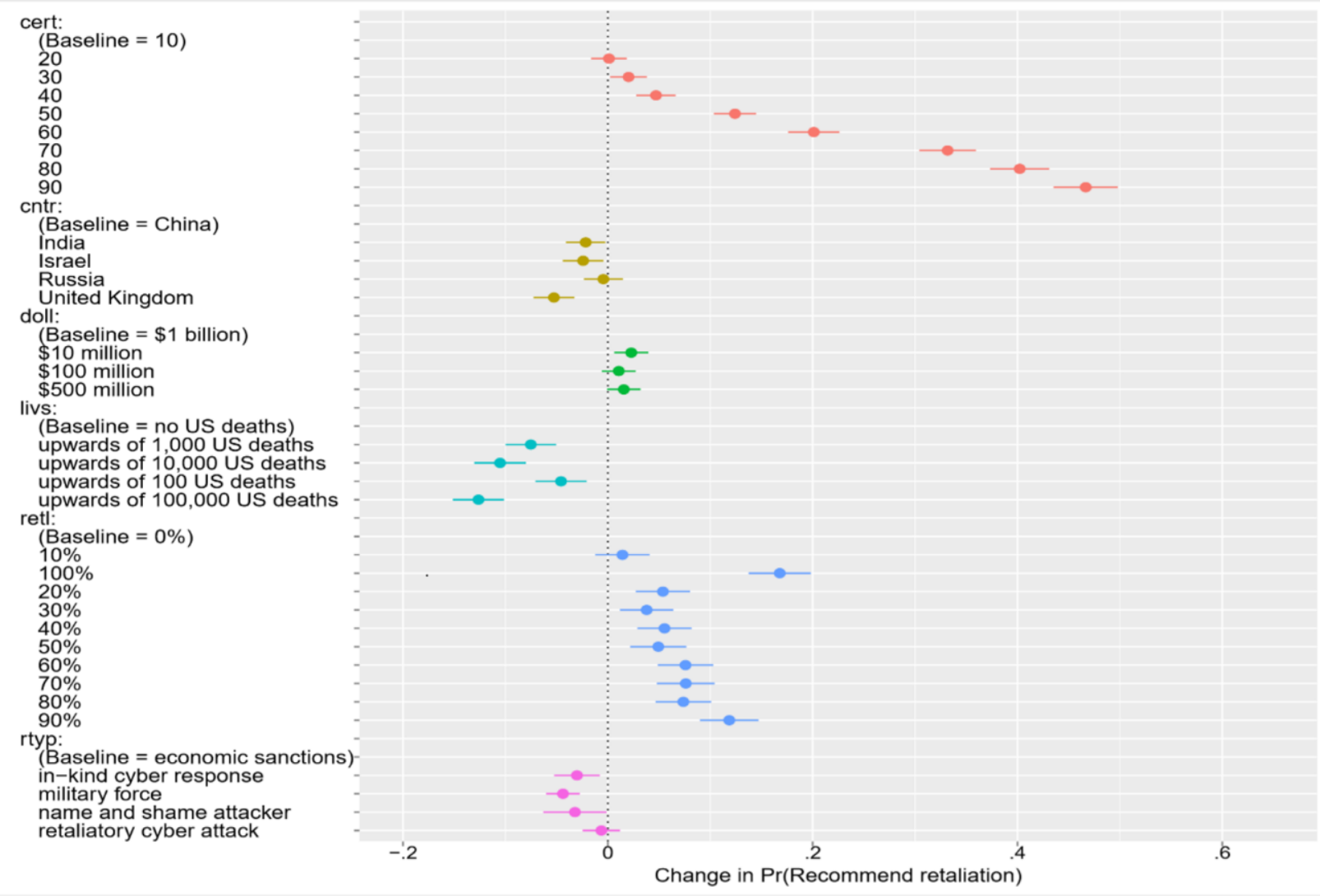

Figure 1. AMCE Plot of Certainty and Probability of Retaliation 
First, there is the lowest certainty grouping. Compared to the 10 percent certainty baseline, the predicted probability that a respondent would choose to retaliate against any erstwhile adversary remains below 10 percent when the government is only 20-40 percent certain about who launched the initial attack against the United States. Beyond these lowest values, there is a modestly discrete jumps in the predicted probability of selecting a retaliatory option as certainty about 'who done it' increases to 50 or 60 percent. When certainty reaches $70-90$ percent, the predicted probability of certainty moves to around 33 percent, 40 percent, and 47 percent, respectively.

No level of certainty results in a predicted probability of retaliation beyond 50 percent. However, compared to other variables in the experimental set up, certainty exerts the largest causal effect on the choice of respondents to retaliate as oppose to doing nothing. For example, even when the probability of another incoming attack hits 100 percent, the predicted probability of choosing to retaliate increases only 17 or 18 percent, well less than half of the effect size for the highest levels of certainty about who launched the initial attack. Similarly, on the suppressive side, the government predicting that retaliation will lead to upwards of 100,000 US deaths only reduces the predicted probability that a respondent will choose to retaliate by 17 percent, roughly equivalent to effect size for the maximal risk of another incoming attack, but still far smaller than the increase in the predicted probability of retaliation due to high levels of certainty about 'who done it.'

\begin{tabular}{|c|c|c|c|c|c|c|}
\hline ertainty & China & India & Israel & Russia & UK & China/UK \\
\hline 10 & 0.074 & 0.059 & 0.075 & 0.055 & 0.058 & 1.270 \\
\hline 20 & 0.068 & 0.076 & 0.082 & 0.068 & 0.042 & 1.644 \\
\hline 30 & 0.072 & 0.090 & 0.082 & 0.110 & 0.071 & 1.022 \\
\hline 40 & 0.171 & 0.088 & 0.099 & 0.105 & 0.115 & 1.484 \\
\hline 50 & 0.226 & 0.193 & 0.202 & 0.216 & 0.110 & 2.059 \\
\hline 60 & 0.294 & 0.239 & 0.223 & 0.319 & 0.244 & 1.205 \\
\hline 70 & 0.398 & 0.417 & 0.385 & 0.440 & 0.344 & 1.157 \\
\hline 80 & 0.506 & 0.474 & 0.482 & 0.466 & 0.422 & 1.199 \\
\hline 90 & 0.563 & 0.531 & 0.524 & 0.556 & 0.487 & 1.156 \\
\hline-10 & 0.152 & 0.134 & 0.127 & 0.161 & 0.051 & \\
\hline-50 & 0.338 & 0.339 & 0.322 & 0.341 & 0.378 & \\
\hline
\end{tabular}

Certainty's effects are also sensitive to pre-existing diplomatic ties. As detailed in Table 1, allies and adversaries have different base rate predicted probabilities of being chosen for retaliation at every level of uncertainty. At the lowest level of certainty, for example, China has a 7.4 percent chance of being selected when identified as the aggressor, while the United Kingdom has only a 5.8 percent predicted probability of being retaliated against. Unsurprisingly, the public is typically more willing to retaliate against historical adversaries than historical allies. Interestingly, at the lowest level of certainty, India and Russia behave like a core US ally, while Israel behaves more like a persistent US adversary. 
Adversarial or alliance ties also affect by how much changing certainty levels matter for the predicted probability that someone will choose retaliation. Clear historical allies, such as the United Kingdom, have a clear reservoir of good will that blunts the effect of increasing certainty up to around the 50 percent level. Potential adversaries such as China do not. For example, the predicted probability that the United Kingdom will be selected as a target of retaliation grows from around 5.8 percent at 10 percent certainty to 10.9 percent at 50 percent certainty - a change of 5.1 percentage points. Movement along the same certainty scale for China results in a much larger amplification effect. China goes from having a predicted probability of being the target of retaliation of 7.4 percent to 22.6 percent, a three-fold increase or a change of 15.1 percentage points. This move can also be seen with the ratio of China to UK predicted probabilities, which is highest at 50 percent certainty. Here, China is over twice as likely to be selected as a target of retaliation than the United Kingdom.

Beyond a positive effect on probability of choosing to retaliate at all, the results from the discrete choice experiment also show that the degree of certainty has a significant effect on the predicted probability of selecting more severe response options. Table 2 breaks the potential retaliatory options into omnibus cost/benefit deciles that reveal the net severity of response options, quantifying the effect of certainty on the predicted probability of selecting increasingly severe countermoves. At the lowest level of certainty (10 percent), the predicted probability of selecting the lowest severity response option is also around 10.7 percent. Holding certainty constant and moving along the row to the most severe retaliatory option lead to a 7.2 percentage point decrease in the predicted probability of selecting the retaliatory choice. At the lowest possible degree of certainty and the highest possible severity of retaliatory option, the predicted probability that the public will choose that course of action is only 3.5 percent.

Table 3. Certainty and Severity of Responses

\begin{tabular}{|c|c|c|c|c|c|c|c|c|c|c|}
\hline \multirow[b]{2}{*}{ Certainty } & \multicolumn{10}{|c|}{ Net Severity (1 = Highest; 10 = Lowest) } \\
\hline & 1 & 2 & 3 & 4 & 5 & 6 & 7 & 8 & 9 & 10 \\
\hline 10 & 0.035 & 0.053 & 0.029 & 0.030 & 0.067 & 0.069 & 0.076 & 0.085 & 0.091 & 0.107 \\
\hline 20 & 0.022 & 0.076 & 0.062 & 0.056 & 0.080 & 0.052 & 0.065 & 0.071 & 0.083 & 0.109 \\
\hline 30 & 0.026 & 0.042 & 0.089 & 0.078 & 0.091 & 0.057 & 0.092 & 0.117 & 0.079 & 0.188 \\
\hline 40 & 0.063 & 0.082 & 0.067 & 0.084 & 0.130 & 0.103 & 0.116 & 0.120 & 0.139 & 0.242 \\
\hline 50 & 0.059 & 0.108 & 0.108 & 0.142 & 0.166 & 0.221 & 0.195 & 0.199 & 0.314 & 0.400 \\
\hline 60 & 0.138 & 0.153 & 0.157 & 0.205 & 0.228 & 0.205 & 0.330 & 0.396 & 0.375 & 0.467 \\
\hline 70 & 0.181 & 0.242 & 0.275 & 0.348 & 0.408 & 0.446 & 0.500 & 0.449 & 0.544 & 0.575 \\
\hline 80 & 0.219 & 0.304 & 0.368 & 0.426 & 0.456 & 0.543 & 0.524 & 0.546 & 0.623 & 0.682 \\
\hline 90 & 0.315 & 0.337 & 0.420 & 0.461 & 0.572 & 0.533 & 0.620 & 0.614 & 0.652 & 0.768 \\
\hline & & & & & & & & & & \\
\hline & 8.961 & 6.306 & 14.539 & 15.432 & 8.530 & 7.683 & 8.160 & 7.189 & 7.133 & 7.152 \\
\hline
\end{tabular}


A similar suppressive move occurs in the top decile of severity. At the lowest level of certainty but the highest severity of the retaliatory options, there is only a 3.5 percent predicted probability that the retaliatory option will be chosen. As certainty increases but the severity of the retaliatory option remains constant, the predicted probability of selecting the choice increases 9-fold, shifting from 3.5 percent at the lowest certainty to 31.5 percent at 90 percent certainty about 'who done it.'

Across the severity scale, the effect of increasing certainty on the selection of a retaliatory option is parabolic, with a maximum at the $4^{\text {th }}$ decile of severity. The lowest severity options tend to be amplified around 7-fold. The highest two severity options also tend to be amplified around 7fold, on average. In the third and fourth highest deciles for severity, maximum certainty about the identity of the perpetrator results in a 14- and 15-fold increase, respectively, in the likelihood of selecting that retaliatory option compared to the lowest certainty levels.

\section{Analysis}

The "attribution problem" is real, but it is also two-sided. Fully specified, the attribution problem predicts that a) deterrence should often fail and b) that escalation from an initial attack to something larger and potentially more damaging should be limited. The results from our experiment confirm these theoretical expectations. A lack of certainty about 'who done it' can inhibit public support for retaliation against an aggressor. Such an inhibition stymies deterrence. If a state is not willing or able to retaliate against a potential aggressor (and the aggressor knows it), deterrence is going to fail $[11,15]$. However, the lack of support for retaliation also expands to cover the public's reticence to select more severe retaliatory options, even after there has been an initial hypothetical attack against the United States. Put otherwise, escalation of conflict in cyberspace is rare because it is not always clear who committed an initial act of aggression.

These results have numerous implications for cyber policy, deterrence posture and efforts to improve the certainty of attribution.

Formulating a deterrent posture in cyberspace requires careful attention to the public's attitudes toward striking back. Better technical, legal, and social processes of attribution can clarify who was likely behind an attack. Entanglement and deterrence by denial can discourage attacks by manipulating the cost-benefit calculus of potential aggressors $[13,14,16]$. Cross-domain deterrence postures can link cyber to the wider panoply of tools at the disposal of government $[23,24]$. The current findings clarify how much certainty is needed for the public to get on side with a retaliation against an aggressor nation. To the extent that elites think like the public [27, 28] or public attitudes shape foreign and defense policy outcomes through public pressure [29], the result can also suggest certain threshold values that matter for actual cyber policy choices and outcomes.

At low levels of certainty, there is a national good-will effect at work. Countries that are historical allies of the United States, such as Great Britain, do not get chosen for retaliation with the same rate of increasing likelihood up to around 50\% certainty. China, a potential adversary of the United States, is rapidly selected as a retaliatory target as the attribution problem subsides. 
These results suggest that the general public weighs in historical alliance ties to a degree when deciding to support retaliation for an attack on the United States. If the government is currently uncertain about 'who done it' and the potential aggressor is an ally, the general public is not likely to be overly supportive. Uncertainty, however, is far less of a shield for historical adversaries.

Efforts to improve attribution, either through technical redesign, political rulemaking, or crossborder data sharing, are a double-edged sword [11,21]. Better attribution will improve a country's deterrence posture, but it will also make public support for and, possibly, the occurrence of escalation more palatable and so more likely. Like cyber deterrence overall [30], the results suggest that the attribution problem is not best managed through simple maximization. Optimization is more prudent. Weathering many low-level incoming cyberattacks because deterrence fails due to attribution problems is likely better than a world of improved attribution wherein incoming attacks have a far higher probability of escalating to something bigger, especially as these escalatory cycles can due to misperception and miscalculation que off wider unanticipated conflicts [31,32]. Assuming the attribution problem can never be completely fixed in cyberspace and so deterrence will always partially fail, balancing the negative and positive effects of uncertainty about 'who done it' can minimize the potential harm and disruption cyberattacks (and responses) can do.

\section{Conclusions}

Certainty matters in cyberspace. It matters because too little disrupts the logic of deterrence, making cyberattacks against the United States more likely. It also matters because too much encourages public support for more severe retaliatory options, effectively increasing the odds that incoming cyber attack will escalate into a wider conflagration. This article has contributed to our understanding of the attribution problem in two ways. First, the discrete choice experiment at the heart of the study quantifies just how much uncertainty about 'who done it' might inhibit public support for retaliation. In other words, the study shows just how much the attribution problem is actually a problem for cyber deterrence. Second, the paper expands and quantifies the theoretical implications of the attribution problem for conflict escalation. The results have numerous implications for the cyber deterrence policies of the United States, wider cybersecurity policy, and ongoing social, technical, and political efforts to ameliorate the attribution problem. Unless deterrence becomes perfectly effective, maintaining some degree of uncertainty can help to suppress more serious conflict and may make everyone better off. 


\section{References}

1 Coburn, A., Leverett, E., and Woo, G.: 'Solving cyber risk : protecting your company and society' (John Wiley \& Sons, Inc.,, 2019. 2019)

2 https://www.us-cert.gov/ncas/alerts/TA18-074A

3 Clarke, R.A., and Knake, R.K.: 'Cyberwar: The Next Threat To National Security and What to do About It' (Harper Collins, 2010. 2010)

$4 \quad$ Kaplan, F.: 'Dark territory: the secret history of cyber war' (Simon and Schuster, 2016. 2016)

$5 \quad$ Rid, T.: 'Cyber war will not take place' (Oxford University Press, 2017. 2017)

6 Lawson, S., and Middleton, M.K.: 'Cyber Pearl Harbor: Analogy, fear, and the framing of cyber security threats in the United States, 1991-2016', 2019, 2019

$7 \quad$ Valeriano, B., and Maness, R.C.: 'Cyber war versus cyber realities : cyber conflict in the international system' (Oxford University Press, 2015. 2015)

8 Jardine, E.: 'Mind the denominator: towards a more effective measurement system for cybersecurity', Journal of Cyber Policy, 2018, 3, (1), pp. 116-139

9 Jardine, E.: 'Global Cyberspace is Safer than you Think: Real Trends in Cybercrime', Global Commission on Internet Governance Paper Series, 2015, (16), pp. 1-22

10 Edwards, B., Hofmeyr, S., and Forrest, S.: 'Hype and heavy tails: A closer look at data breaches', Journal of Cybersecurity, 2016, 2, (1), pp. 3-14

11 Buchanan, B.: 'Attributing Cyber Attacks AU - Rid, Thomas', Journal of Strategic Studies, 2015, 38, (1-2), pp. 4-37

12 Brantly, A.F.: 'DECISION TO ATTACK : military and intelligence cyber decision-making' (UNIV OF GEORGIA PRESS, 2018. 2018)

13 Brantly, A.F.: 'The cyber deterrence problem', in Editor (Ed.)^(Eds.): 'Book The cyber deterrence problem' (2018, edn.), pp. 31-54

14 Brantly, A.: 'Conceptualizing Cyber Deterrence by Entanglement', in Editor (Ed.)^(Eds.): 'Book Conceptualizing Cyber Deterrence by Entanglement' (2018, edn.), pp. 18

15 Clark, D.D., and Landau, S.: 'Untangling attribution', Harv. Nat'I Sec. J., 2011, 2, pp. 323

16 Nye, J.S.: 'Deterrence and Dissuasion in Cyberspace', International Security, 2017, 41, (3), pp. 44-

71

17 Lindsay, J.R.: 'Tipping the scales: the attribution problem and the feasibility of deterrence against cyberattack', Journal of Cybersecurity, 2015, 1, (1), pp. 53-67

18 Schelling, T.C.: 'The Strategy of Conflict', 2015

19 Schelling, T.: 'Arms and influence' (Green Wood., 1966. 1966)

20 Maurer, T.: 'Cyber mercenaries : the state, hackers, and power' (Cambridge University Press, 2018. 2018)

21 Bradshaw, S., Raymond, M., and Shull, A.: 'Rule Making for State Conduct in the Attribution of Cyber Attacks', Mutual Security in the Asia Pacific: Roles for Australia, Canada, and South Korea, 2015, pp. $125-143$

22 Gartzke, E., and Linsday, J.: 'Cross-Domain Deterrence: Strategy in an Era of Complexity'. Proc. International Studies Association, Toronto, CA2014 pp. Pages

23 Gartzke, E., and Lindsay, J.R.: 'Cross-Domain Deterrence: Strategy in an Era of Complexity' (Oxford University Press, 2019. 2019)

24 Lindsay, J.R., and Gartzke, E.: 'Cross-domain deterrence as a practical problem and a theoretical concept', Cross-Domain Deterrence: Strategy in an Era of Complexity, Gartzke E and Lindsay JR (eds.), La Jolla, CA: Manuscript, 2016

25 Hainmueller, J., Hangartner, D., and Yamamoto, T.: 'Validating vignette and conjoint survey experiments against real-world behavior', Proceedings of the National Academy of Sciences, 2015, 112, (8), pp. 2395 
26 Hensher, D.A., Rose, J.M., and Greene, W.H.: 'Applied choice analysis: a primer' (Cambridge University Press, 2005. 2005)

27 DeNardo, J.: 'The amateur strategist: Intuitive deterrence theories and the politics of the nuclear arms race' (Cambridge University Press, 1995. 1995)

28 Herron, K.G., and Jenkins-Smith, H.C.: 'US perceptions of nuclear security in the wake of the Cold War: Comparing public and elite belief systems', International Studies Quarterly, 2002, 46, (4), pp. 451-

479

29 Tomz, M.R., and Weeks, J.L.: 'Public opinion and the democratic peace', American political science review, 2013, 107, (4), pp. 849-865

30 Jardine, E.: 'Optimizing Cyber Deterrence', in Brantly, A. (Ed.): 'The Cyber Deterrence Problem' (Roman \& Littlefield, 2020)

31 Jervis, R.: 'Perception and misperception in international politics : with a new preface by the author', 2017

32 Jervis, R.: 'Hypotheses on Misperception', World Politics, 1968, 20, (3), pp. 454-479 